\title{
TUBERCULOSIS OF THE LIP*
}

ERWIN P. ZEISLER, M.D.

Attending Dermatologist, Cook County Hospital and Wesley Memorial Hospital

CHICAGO

Tuberculosis in the buccal cavity presents itself in almost as many diverse forms as on the integument, and frequently gives rise to diagnostic errors. The subject is given scant consideration in textbooks on dermatology, usually under the heading of tuberculosis cutis orificialis, and in books on general medicine it is considered briefly in relation to respiratory tuberculosis. Special treatises on diseases of the mouth, such as that of Mikulicz and Kümmell, ${ }^{1}$ recognize these types of oral tuberculosis: (1) lupus of the mucous membranes; (2) tuberculosis ulcerations, rhagades and infiltrates of the tongue and lip; (3) isolated ulcers of the soft palate; (4) miliary ulcerations occurring at the corners of the mouth, on the lip and edges of the tongue in advanced cases of phthisis. Jadassohn, ${ }^{2}$ in his masterly review of the subject of tuberculosis of the skin and mucous membranes, divides this into: (1) lupus of the mucous membranes; (2) miliary ulcerations involving the mucous membranes and adjacent skin ; (3) tuberculous ulcers of nonmiliary character involving the lip, tongue and genital regions. Osler ${ }^{3}$ states that tuberculosis of the lip is extremely rare even in cases of advanced pulmonary disease. Considering the frequency with which tubercle bacilli in the sputum come in contact with the lips, it seems unusual that they should be so seldom affected. An internist who sees large numbers of pulmonary cases recently stated that he had never seen an involvement of the lip in more than 20,000 patients with tuberculosis examined by him. In view of these facts, the subjoined case seems of sufficient interest to warrant a detailed report.

* From the Department of Dermatology and Syphilology, Northwestern University Medical School.

1. Mikulicz, J., and Kümmell, W.: Erkrankungen der Mundhöhle, Ed. 2, Jena, 1911.

2. Jadassohn, J.: Tuberkulose der Haut, Mracek's Handbuch der Hautkrankheiten 4:1, 1907.

3. Osler, William: Practice of Medicine, Ed. 8, New York, D. Appleton \& Co., 1912, p. 211. 


\section{REPORT OF CASE}

History--M. H., man, aged 56, presented himself at the Northwestern Medical School Dispensary complaining of a painful swelling of the lower lip. He gave a history of repeated attacks of pneumonia, the first one in 1890 in Russia, and again in 1902. Ten years ago, he had bronchitis and pleurisy. For many years he had had a winter cough, which had stopped three years before when he discontinued smoking. Recently he had had occasional night sweats, and had lost some weight. There was no history of hemoptysis. Ten months ago he first noticed the swelling of the lip which grew worse after influenzal pneumonia of three weeks' duration, in May, 1919. About the same time, ulcers in the mouth developed, causing pain and a burning sensation and difficulty in eating. He had had several teeth extracted in the last few months. His appetite was poor, and he complained of general weakness.

The family history was uneventful. Four sisters and one brother were living in Russia. His father died at the age of 68 of paralysis, and his mother

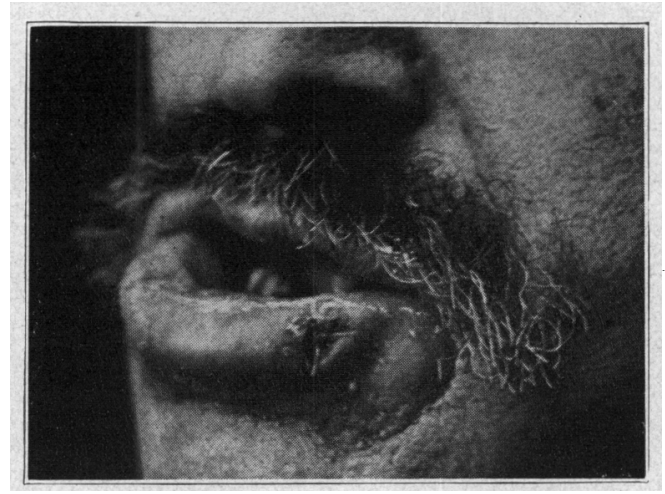

Fig. 1.-Tuberculosis of the lip. (The ulceration at the right is the biopsy wound.)

at 76 of senility. There was no family history of tuberculosis. One daughter and one son were in good health.

Physical Examination.-The patient was extremely emaciated and pale. Except for the chest findings, the physical examination was negative. Dr. Herbert W. Gray of the medical department made a careful examination of the chest and reported as follows: Distinct drooping of the right shoulder; very prominent clavicles on both sides, especially the right, on which side the fossae are very prominent; dull on percussion over both apexes and over the first left interspace; hyperresonant over the second interspace and dull over the third and fourth on the same side; dull along both sides of the sternum from the first to the fourth interspaces.

Heart examination revealed the apex in the sixth interspace in the midclavicular line. The right border was $1.5 \mathrm{~cm}$. left of the left parasternal line. There were two tones but no murmurs. Posteriorly the thorax was dull and hyperresonant, bilaterally from the apexes down to the eighth rib and laterally to the midscapular line on the right and to the inferior border of the scapula on the left. The rest of the chest, particularly the right anterior and both 
axillary regions, and inferiorly below the eighth ribs, was distinctly hyperresonant to percussion with diminished breath sounds. There was compensatory breathing over both lower lobes in the axillary area. Distinct bronchial breathing of a cavernous character was noted at the apex of the right upper lobe. There was a difference of $1 \mathrm{~cm}$. letween forced inspiration and expiration, showing the high grade of emphysema present in the functioning lung. No ràles were heard during the entire examination.

Diagnosis.-The diagnosis was chronic phthisis, with extensive chronic emphysema; heart displaced to the left due to pulling of adhesions; chronic mediastinitis: very slight degree of activity at the present time.

Rocutgcnologic Findinys.-Dr. Blumenthal of the roentgen-ray department reported on the fluoroscopic examination as follows; extensire fibrosis of upper right lung; pericardial adhesions to the diaphragm; adhesions to the trachea displacing it toward the right; moderate fibrosis of the left lung.

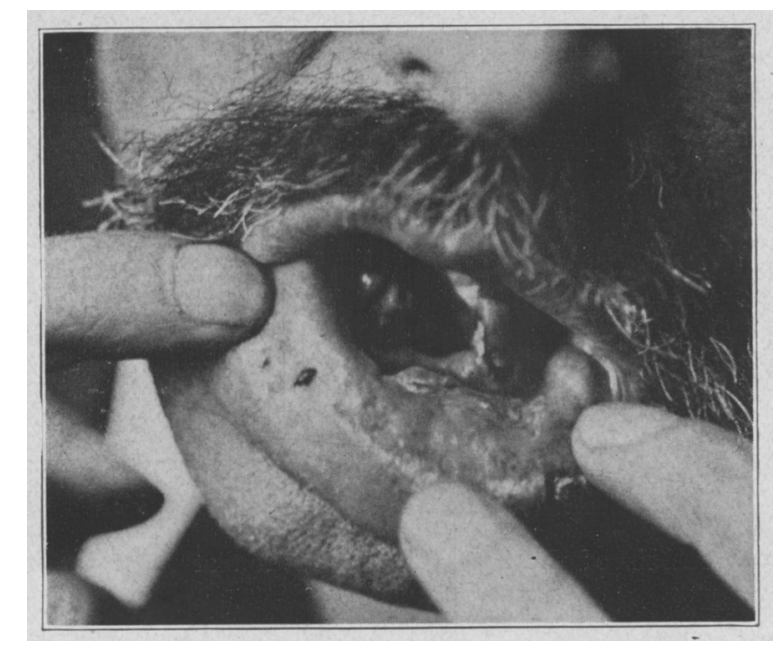

Fig. 2.-Tuberculosis of the lip showing ulceration of the buccal mucous membrane.

Description of Lcsions in the Mouth.-The entire lower lip was uniformly enlarged, about twice the size of the upper lip, and indurated to the touch, with several small areas of denuded epidermis. Under glass pressure, minute nodules could be discerned. The inner aspect of the lower lip down to the gingival border showed a superficially ulcerated area about $2 \mathrm{~cm}$. broad with a granular base, sloping edges and was surrounded by edematous mucous membrane for some distance on each side. On palpation, the ulcer felt moderately indurated. At the inner aspect of the upper lip near the right commissure was an abruptly excavated ulcer $0.5 \mathrm{~cm}$. in depth with an irregular base. This ulcer felt soft to the touch. Opposite the last lower right molar was a third shallow ulcer about $1 \mathrm{~cm}$. in circumference of a similar type. The few remaining teeth in the lower set showed evidence of extensive decay and pyorrhea. The gums of the lower jaw were spongy, edematous, and exuded pus on pressure. The tongue, palate and pharynx appeared normal. A laryngologic examination was negative. 
Laboratory Findings.-The Wassermann reaction was negative. The blood pressure was 115 systolic, and 65 diastolic. Hemoglobin was 75 (Sahli). Red blood cell count revealed 5,200,000 cells; the leukocyte count was 6,400. Differential count demonstrated: neutrophils 71 per cent., small mononuclears 22 per cent., large mononuclears 6 per cent., transitionals 2 per cent., basophils 1 per cent, eosinophils none. Urine examination disclosed: acid, specific gravity 1.018 , faint trace of albumin, no sugar and no acetone. Microscopically, occasional hyaline casts were seen.

Diagnostic Comment.-The patient was exhibited at one of the meetings of the Chicago Dermatological Society, and the majority of the members expressed the opinion that the lesion of the lower lip was an epithelioma. The patient's age, the induration and the presence of a hard mass of glands in the submental region favored this diagnosis. However, the presence of a second ulceration near the angle of the mouth separated by an area of normal mucous membrane and of a third ulcer of the buccal mucosa was not entirely consistent with this view. Smears and scrapings were made from the ulcers in the mouth, stained with Ziehl's stain and showed large numbers of acid-fast bacilli-short thin rods of granular type, morphologically identical with tubercle bacilli. The diagnosis of tuberculosis of the lip with tuberculous ulcers of the mouth was further substantiated by the results of the biopsy. What appeared to be merely an edema of the lip proved to be a solid tuberculoma.

Histologic Examination.-Two pieces of tissue were removed for histologic study, one from the ulcer of the mouth and a wedge-shaped piece of tissue from the vermilion border of the lip. In addition to the usual stains, special stains for acid-fast bacilli were made. The lesion from the lip showed the epidermis to be normal or slightly thinned in part of the section, with the stratum granulosum intact. In other areas, there was extreme acanthosis, irregular down-growth of rete pegs, intracellular and extracellular edema of rete cells and infiltration with leukocytes.

The corium showed intense edema and dilatation of lymphatics and capillaries. Numerous cell masses were present in the middle and subpapillary layers. The cellular infiltration was composed of isolated and conglomerate tubercles of varying size with chanacteristic arrangement of giant cells, epithelioid cells and small round cells. Giant cells were numerous, oval or round, with ten to twenty peripherally arranged nuclei corresponding to Langhans' type. There was a tendency to conglomeration of tubercles around the vascular channels with an inflammatory zone around the cell groups. There was no tendency to coagulative necrosis of the center of the tubercles as seen in lupus. The collagen was edematous and stained poorly with eosin. The blood vessels were dilated with swollen endothelium. Ziehl's stain showed scanty numbers of tubercle bacilli in the corium. The histologic examination of the lesion from the floor of the mouth showed absence of the epidermis, a diffuse cellular invasion of the corium with relatively few giant cells and no definite arrangement into tubercles, but large numbers of acid-fast bacilli, many of them in clumps.

Treatment and Subsequent Course.-The patient was placed on a general tuberculous regimen, a high protein diet and cod-liver oil internally. Locally a cocain ointment was used on the lip to relieve the pain, and the ulcers in the mouth were swabbed several times daily with diluted hydrogen peroxid. Filtered Coolidge treatments were administered over the lip and submental region. In 
the course of six weeks, the swelling of the lip had diminished appreciably in size, and the ulcerations in the mouth had healed. The patient had gained in weight, but his general condition was still poor, and after several months he disappeared from observation.

GENERAL REITEW OF THE SLISJET OL TLBERCLLOSIS OF THE LIP

The occurrence of tuberculous ulcers of the mouth and lips of the miliary type in adranced cases of phthisis has been known for a long time through the observations of Jarisch, ${ }^{4}$ Chiari" and especially Kaposi." Among more recent reports of cases involving the lips are those of I'lichon," Schuchardt," Morestin." Thibierge. Hallopean and

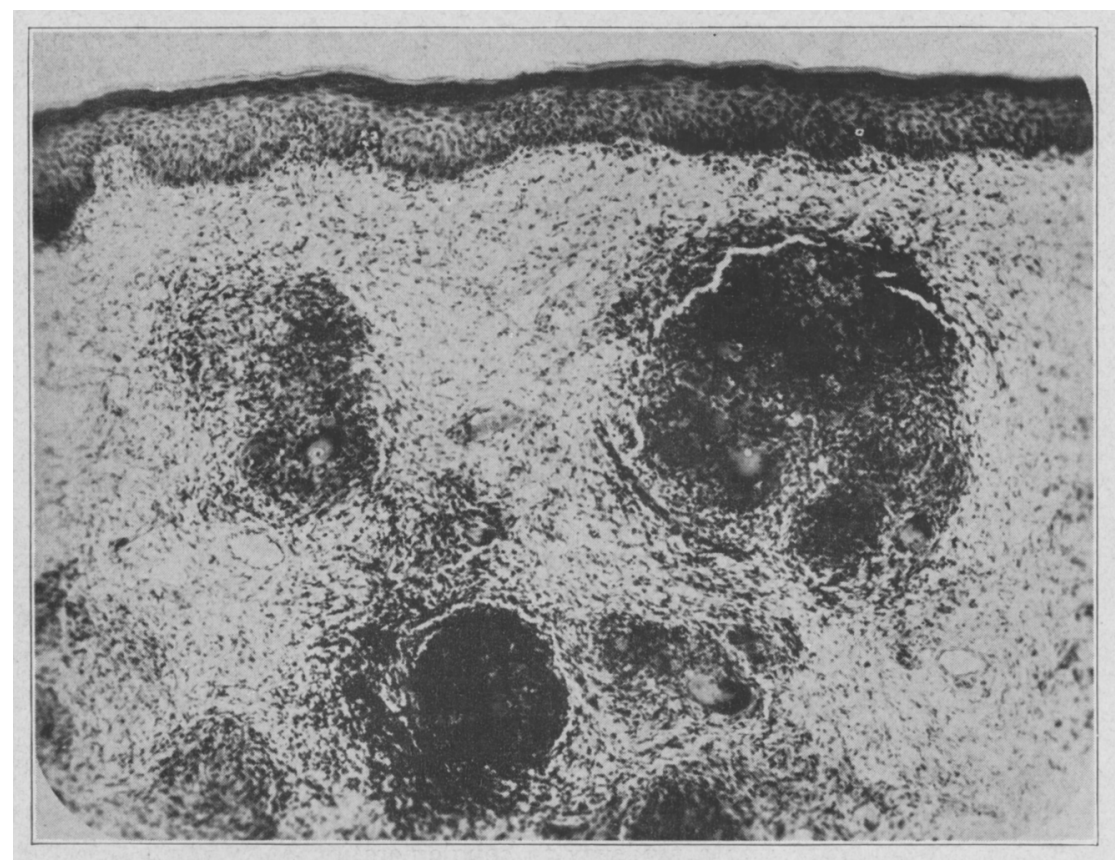

Fig. 3.-Low power photomicrograph showing isolated tubercles in the corium.

Ribot," Münter ${ }^{12}$ and others. These cases have been reported under various titles, as tuberculosis cutis vera, tuberculosis ulcerosa. tuber-

4. Jarisch: Arch. f. Dermat. u. Syph.. 1879, p. 265.

5. Chiari: Arch. f. Dermat. u. Syph., 1879, p. 269.

6. Kaposi: Arch. f. Dermat. u. Syph. 43:373, 1898.

7. Plichon. M.-E.: Tulerculose des lèvres. Paris, 1888.

8. Schuchardt: Deutsch. med. Wchnschr. 15:1073, 1889.

9. Morestin: Bull. et mém. Soc. anat. de Paris 79:108. 1904.

10. Thibièrge: Amn. de dermat. et syph. 8:286, 1897.

11. Hallopeau and Ribot: Ann, de dermat. et syph. 13:611, 1902.

12. Münter: Berl. klin. Wchnschr. 49:1495, 1912. 
culosis cutis orificialis and tuberculosis miliaris. The disease is confined to the mucous membrane and skin about the mucous outlets. Kaposi, in his series of twenty-two cases, found the cutaneous part of the lip involved in eight and the mucosa in six. The clinical characteristics of tuberculous ulcers of the miliary type are well known. They are shallow, round or oval in outline, with a pale red base covered with a seroviscid secretion and irregular borders showing miliary nodules. Tuberculous ulcers of the mouth and lips are further characterized by their rapid development, occasional destructive tendency and acute pain. It is rare to see the grouped miliary nodules before they break down. Ulcers form in from three to eight days and spread either deeply or superficially, simulating the breaking down of a gumma or carcinoma. The prognosis is usually grave as most patients are in the terminal stages of tuberculosis. Kaposi found that the outlook was not hopeless in every case, as the ulcers may show a tendency to heal after a certain stage is reached.

Tuberculous ulcers of the mucous membrane of nonmiliary type are not so well known and have been emphasized especially by Jadassohn, ${ }^{2}$ Miyahara, ${ }^{13}$ Danlos ${ }^{14}$ and others. They are atypical, torpid, poorly granulating papillary ulcerations with undermined or heapedup borders and resemble lupus ulcers. They are more chronic in their course and occur in association with visceral tuberculosis of a less fulminating type. A special variety is called by Jadassohn the chancriform or epitheliomaform type on account of its firmer consistency and the close resemblance to chancre or epithelioma. These tuberculous lesions occur on the lips as the result of exogenous inoculation in certain instances, and are analogous to the tuberculous ulcers of the genitalia, resulting from infection through ritual circumcision, and those around the fingers and nails occurring as a result of accidental inoculation from tuberculous material. In two cases of lesions on the lower lip, Jadassohn was not able to exclude the diagnosis of epithelioma until the histology was studied and tubercle bacilli were found. Miyahara has reported in detail a lesion on the vermilion border of the lower lip in the form of an indurated plaque, $1.5 \mathrm{~cm}$. in diameter. Histologically he found small, sharply defined tubercles in the upper corium along the vein walls and around the lymphatics. Bacillary findings as well as guinea-pig inoculations were positive. A nodule in the cheek also showed a tuberculous structure and was regarded as a lymphogenous metastasis. There were no tuberculous lesions demonstrable elsewhere in the body, and the case was regarded as probably

13. Miyahara: Arch. f. Dermat. u. Syph. 111:305, 1912.

14. Danlos: Bull. Soc. franç. de dermat. et syph. 10:12, 1899. 
the result of exogenous inoculation. The second case was a chancriform tuberculosis of the lip in a child of 9 years. On the middle of the upper lip was a crust-covered ulcer, 0.5 by $3.25 \mathrm{~cm}$., with some infiltration. Excision showed tuberculous structure with sparse bacilli. Later the child died of pulmonary and meningeal tuberculosis. Danlos, Münter and others have also reported tuberculous ulcers of the lip which resembled chancre. Many of the cases reported in the literature (Fleming, Bamberger, Paget, Spitzer, etc.) have been mistaken for epithelioma on account of the firm consistency of the border and base and the granular surface of the ulceration. The case here reported resembled more the epitheliomaform type of Jadassohn and was rather unusual in that it represented a solid tuberculoma of the entire lower lip with ulcerations of the buccal mucosa.

The pathogenesis of tuberculous lesions of the lip as well as other portions of the mucous membrane presents several possibilities. There may be a local infection of the lip from without, as in Miyahara's case, and a dissemination to other organs from this source.. A rare possibility is a simultaneous infection of the mucous membrane and other viscera. The most probable mode of infection is a primary respiratory infection with secondary involvement of the lip by autoinoculation or hematogenous infection. The lesion of the lip represents in most instances a direct infection of the tissues through a fissure or break in the mucous membrane. In the case here reported, the presence of carious teeth and infected gums in the lower jaw gave a clue to the possible mode of entrance of the bacilli. The extraction of teeth has been known to be followed by mucous membrane tuberculosis and involvement of the alveolar process and regional lymph glands.

The diagnosis of tuberculosis of the lip rests largely on the clinical characteristics of the ulcers, the absence of the cartilaginous induration of most chancres and epitheliomas, the negative spirochete examination and the presence of acid-fast bacilli in smears or scrapings. When bacilli are not found, a biopsy will settle the diagnosis. In the miliary ulcerations the histologic picture will show chronic granulation tissue with few giant cells and an abundance of bacilli. The nonmiliary type has a histology resembling Boeck's miliary lupoid with sharply defined tubercles in the middle and upper corium with bacilli in scanty numbers. The typical arrangement of central giant cells, epithelioid and small round cells is found in these cases, whereas cheesy degeneration is absent. Guinea-pig inoculations may also be made in doubtful cases.

The treatment of tuberculous ulcers of the mouth includes the general treatment of the visceral infection, local use of antiseptic mouth 
washes and local anesthetics for the relief of pain. Fifty per cent. lactic acid has been recommended as a cauterizing agent. Curetting or excision might be resorted to in favorable cases. The roentgenray and Finsen light have also been used. The prognosis depends on the extent of the visceral involvement.

\section{CONCLUSIONS}

1. The case here reported represents an unusual instance of solid tuberculoma of the lower lip with ulcerations of the buccal mucosa associated with a chronic pulmonary tuberculosis of the fibroid type.

2. Tuberculosis of the lip must be differentiated from chancre and epitheliomatous ulcerations.

3. A positive diagnosis rests on the finding of tubercle bacilli and the characteristic histology. 